\title{
Synchronous Collaborative Navigation on the WWW
}

\author{
Yann Laurillau \\ Laboratoire CLIPS/IMAG \\ BP 53, 38041 Grenoble Cedex 9, FRANCE \\ +33476514440 \\ Yann.Laurillau@imag.fr
}

\begin{abstract}
We describe an ongoing research effort in designing synchronous collaborative navigational techniques on the WWW. The goal of the research program is to provide usable tools to collaboratively search information on the WWW. We begin by studying collaborative navigational techniques in the search results space defined by a WWW search engine. We first present our design method. We then characterize four types of collaborative navigation that are embedded in the current prototype, Co-Vitesse.
\end{abstract}

\section{Keywords}

Collaborative/Social Navigation, CSCW, Design Method.

\section{INTRODUCTION}

Seeking information through the World Wide Web is difficult. Users are "lost in hyperspace" [1] and have difficulties "in gaining an overview of the material" [3]. In addition, navigational behavior on the web often relies on the expertise of other users. One typical social behavior on the web consists of asking a colleague about information we assume the other has the pointer to: Such observed social behaviors show that web users are striving for collaboration. Several collaborative tools, including chat room, mail or news for exchanging URLs [2], can help the users in their information retrieval but are not integrated in browsers. Moreover most of these tools only support asynchronous collaboration.

Our research program consists of designing and building synchronous collaborative tools for seeking information on the web. Indeed, as motivated above, the need for systems on the web that support coordination, communication and shared production between and within groups increases markedly. We begin by focusing on synchronous navigation in the search results space: Such tools are dedicated to a group of users searching information. Examples of usage include:

- A group of master students have to write a bibliography about a particular subject.

- A group of workers are responsible for monitoring technological development in a firm.

\section{DESIGN METHOD}

As shown in Figure 1, our design method is based on a conceptual study of navigational tasks as well as

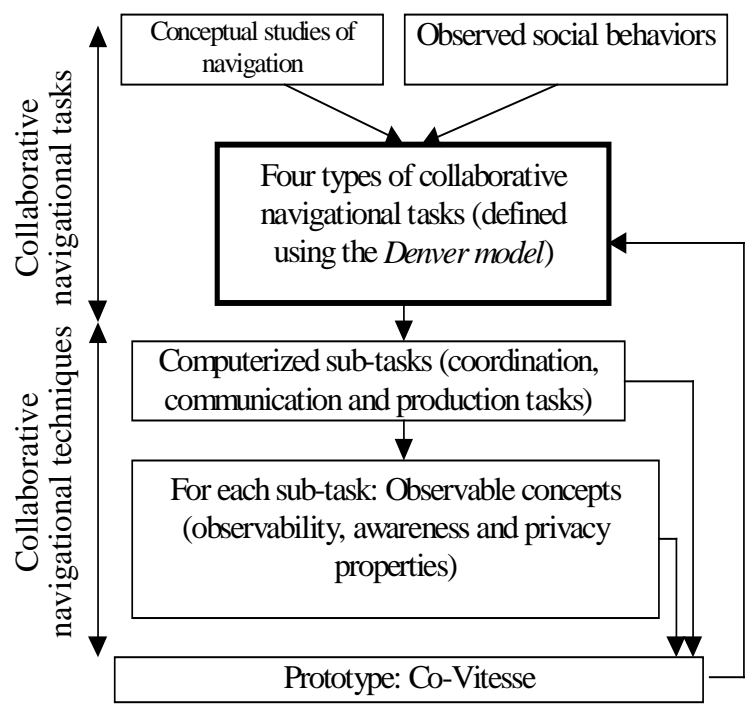

Figure 1: Design method.

observed social behavior of users while searching for information. The results of these studies and observations enable us to identify four types of collaborative navigational tasks that are described in the following paragraph. In the next step, we refine the four types of navigation in terms of sub-tasks that are organized according to the three spaces: communication, coordination and production spaces. We then address the awareness and privacy issues by studying relevant concepts to be made observable by the users for each task.

\section{COLLABORATIVE NAVIGATIONAL TASKS}

Our work uses the observed collaborative activities described in [6]. In that study, work-related collaborations between users of OPAC terminals in Lancaster University Library were observed. From these observed social behaviors, we identify four types of navigational tasks by applying the Denver model [5], which involves characterizing the interactive situations and protocols of each navigational task:

- Relaxed navigation: It defines an open group without a leader. No objective is clearly defined and it is completely informal. Users navigate independently. Nevertheless at any time the user can give the control to someone else if she/he needs help.

- Cooperative navigation: There is again no leader, but members have a common objective that was 
planned in advance. Each member has to explore a predefined section of the space. All the members can accept or decline a newcomer. Each member automatically receives the current findings of the group (marked web pages) when leaving the group.

- Planned navigation: The leader decides the objective of the session and has the responsibility for assigning a section of the shared information space to each member. The member can individually work on her/his assigned section until the leader decides to gather the findings of all the members. In addition the leader can group together (" teleport") all the members in a particular location of interest for the group.

- Guided tour: The guide freely navigates and the members follow the guide. A user can join a guided tour at any time. The guide is by definition an expert of the information space and/or the domain.

These four types of navigation are implemented in the Co-Vitesse system that is described below.

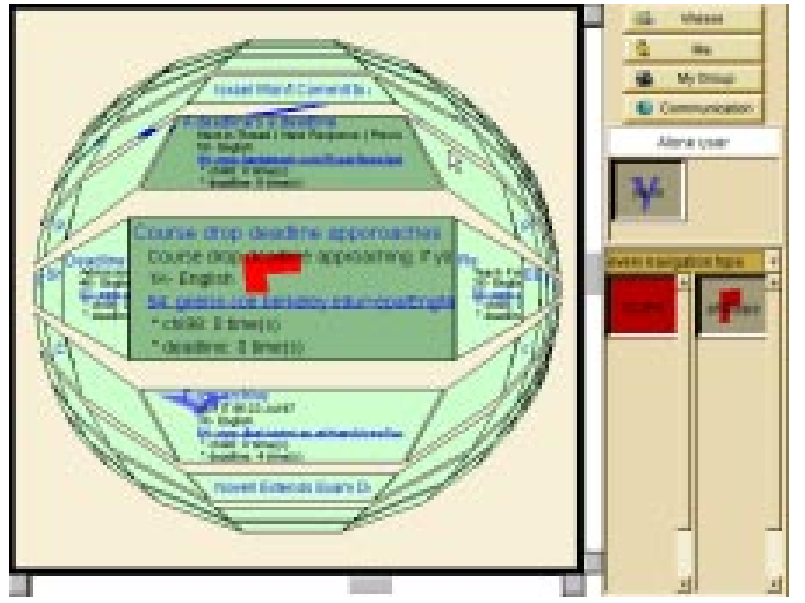

Figure 2: A snapshot from the Co-Vitesse system: Main window.

\section{APPLICATION}

The Co-Vitesse system enables the users to navigate synchronously on the WWW. The four types of navigational tasks are explicitly available to the users. Co-Vitesse is based on a single-user application, Vitesse [4]. The VITESSE system visualizes the results of a query submitted to a search engine on the WWW. As shown in Figure 2 (left part), the overall graph structure of the results is displayed (six visualization techniques are available): Each retrieved page (node) and their links are displayed. One retrieved page or node is displayed by a polygon. The selection of a node (double click) enables the user to access the web page.

When starting a session, a Co-Vitesse user defines his/her avatar by a shape and a name. The user then either selects an information space or specifies a query that will be sent to a selected search engine. The results of the query define a new information space. The user

can then navigate in the information space, observe other users (in Figure 2, two users are navigating), create or join a group.
On the right side of the space, two palettes are displayed. One palette displays all the single users and the groups in the information space. Selecting a group will make the corresponding members observable on the information space and in the second palette. The user can then opt to only observe some of the members of the group by selecting their corresponding icons. A group is represented by a color and a name. If a user belongs to a group, her/his shape will be displayed in the color of the corresponding group; else a predefined color is automatically assigned to a user.

Additional windows are available through the menu in the top right corner. The windows are organized according to three sets of tasks: single-user tasks, group tasks and communication tasks (chat room). One of the main single-user tasks is the creation of a group: At any time, a single user can create a group, its objective and its style of collaborative work by selecting one of the four types of navigation.

At the end of the session each user collects the findings of the group gathered in his/her bookmark.

\section{CONCLUSION}

The next step in our work is to experimentally test CoVitesse in order to evaluate the four types of navigation. We plan to ask a group of master students of our University to perform scenarios while having wizards serving as guides (guided tour) and leaders (planned navigation).

\section{ACKNOWLEDGMENTS}

This work is supported by the French Ministers of Research and Industry Contract SIRII. Many Thanks to L. Nigay my Ph.D. supervisor, F. Vernier and P. Renevier for their help in developing the Vitesse and Co-Vitesse systems.

\section{REFERENCES}

1. Conklin, J. Hypertext : an introduction and survey. IEEE Computer, 20(9), September 1987, 17-41

2. Dieberger, A. Supporting social navigation on the World Wide Web. International Journal of HumanComputer Studies, 46, 1997, 805-825.

3. Hammond, N., Allinson, L. Extending Hypertext for Learning : An investigation of Access and Guidance Tools, in Proceedings of HCI'89 (Nottingham, Sept. 1989), Cambridge University Press, 293-304.

4. Nigay, L., Vernier, F. Design Method of Interaction Techniques For Large Information Spaces, in Proceedings of AVI'98 (Aquila, May 1998), ACM Press, 37-46.0

5. Salvador, T., Scholtz, J., Larson, J. The Denver Model for Groupware Design. SIGCHI Bulletin, 28(1), Jan. 1996.

6. Twidale, M., Nichols, D., Paice, C. Browsing is a collaborative process. Information Processing \& Management, 33(6), 1997, 761-83. 